\title{
Minimizing Financial Cost of Scientific Workflows Under Deadline Constraints in Multi-Cloud Environments
}

\author{
Tianyu Gao \\ School of Information Science and \\ Technology, Northwest University \\ Xi'an, Shaanxi, China 710127 \\ gaotianyu@stumail.nwu.edu.cn \\ Yongqiang Wang \\ School of Information Science and \\ Technology, Northwest University \\ Xi'an, Shaanxi, China 710127 \\ yqwang@nwu.edu.cn
}

\author{
Chase Q. Wu \\ Department of Computer Science, \\ New Jersey Institute of Technology \\ Newark, New Jersey, USA 07102 \\ chase.wu@njit.edu \\ Ruxia Li \\ School of Information Science and \\ Technology, Northwest University \\ Xi'an, Shaanxi, China 710127 \\ liruxia@stumail.nwu.edu.cn
}

\author{
Aiqin Hou \\ School of Information Science and \\ Technology, Northwest University \\ Xi'an, Shaanxi, China 710127 \\ houaiqin@nwu.edu.cn \\ Mingrui Xu \\ School of Information Science and \\ Technology, Northwest University \\ Xi'an, Shaanxi, China 710127 \\ airkirax@stumail.nwu.edu.cn
}

\begin{abstract}
In recent years, cloud platforms have been rapidly developed and deployed around the globe and many large-scale scientific workflows have been migrated to multiple clouds for cost-effective data analysis. In such cloud-based workflow applications, financial cost is a major concern in addition to traditional performance requirements such as execution time. In this paper, we formulate a workflow mapping problem to minimize the financial cost of deadlineconstrained scientific workflows executed in multi-cloud environments, referred to as MinCost-MC, which is shown to be NP-complete. Within a generic three-layer workflow execution framework, we propose a Workflow Mapping algorithm for Financial Cost Optimization, referred to as WMFCO. This algorithm takes in consideration storage requirements, $I / O$ operations, and data transfers to minimize the financial cost of a given workflow within a specified deadline. Extensive simulation results show that WMFCO exhibits a superior performance over existing algorithms in terms of financial cost in multi-cloud environments.
\end{abstract}

\section{CCS CONCEPTS}

- Applied computing; • General and reference $\rightarrow$ Performance; Reliability;

\section{KEYWORDS}

Workflow mapping, cloud computing, cost optimization

\section{ACM Reference Format:}

Tianyu Gao, Chase Q. Wu, Aiqin Hou, Yongqiang Wang, Ruxia Li, and Mingrui Xu. 2019. Minimizing Financial Cost of Scientific Workflows Under Deadline Constraints in Multi-Cloud Environments. In The 34th ACM/SIGAPP Symposium on Applied Computing (SAC'19), April 8-12, 2019, Limassol, Cyprus. ACM, New York, NY, USA, 8 pages. https://doi.org/10.1145/3297280.3297293

Permission to make digital or hard copies of all or part of this work for personal or classroom use is granted without fee provided that copies are not made or distributed for profit or commercial advantage and that copies bear this notice and the full citation on the first page. Copyrights for components of this work owned by others than ACM must be honored. Abstracting with credit is permitted. To copy otherwise, or republish, to post on servers or to redistribute to lists, requires prior specific permission and/or a fee. Request permissions from permissions@acm.org.

SAC'19, April 8-12, 2019, Limassol, Cyprus

(C) 2019 Association for Computing Machinery.

ACM ISBN 978-1-4503-5933-7/19/04 . \$ \$15.00

https://doi.org/10.1145/3297280.3297293

\section{INTRODUCTION}

An increasing number of scientific applications are using workflow technologies to manage and execute the process of data generation, synthesis, visualization, and analysis for scientfific innovation and knowledge discovery. To some degree, scientific workflows have become an indispensable component of the research infrastructure in a wide spectrum of science domains.

Many scientific workflows routinely process a tremendous amount of data, which requires computing power far beyond any individual computer. Due to the economic benefits and storage services provided by cloud computing, scientific workflows, especially those of large scales, have been actively migrated to clouds for cost-effective data analysis. There exist a significant number of efforts on workflow mapping and scheduling to meet traditional performance requirements such as execution time. In fact, some scientific computations must be completed in a timely manner to produce valuable results, such as hurricane prediction or weather forecast in general. However, when executed in cloud environments, financial cost has become another important concern for workflow optimization.

As widely deployed, there are three types of common services in cloud computing: Software as a Service (SaaS), Platform as a Service (PaaS), and Infrastructure as a Service (IaaS). Among them, IaaS clouds provide virtual machines (VMs) with customizable computing power to satisfy various needs of different applications, and hence are well suited for executing scientific workflows. One typical example is the Amazon EC2[1] using IaaS cloud service. In IaaS cloud service, the price of VMs is generally based on their performance parameters[17] per time unit that service providers specify, usually in hours. For workflow execution in a single cloud, the data transfer time typically accounts for less than 10 percent of the overall execution time, and hence the cost of data transfer is oftentimes ignored in most existing research. However, with an explosive increase in the data volume, multi-cloud environments have become a necessity for many large-scale scientific workflows. In such environments, the time and cost of inter-cloud data transfer may be comparable to those of the computing task, and should be explicitly accounted for. In addition, big data poses a significant challenge to storage, which has not been well investigated in most existing efforts for workflow optimization. 
In this paper, we formulate a workflow mapping problem to minimize the financial cost of a deadline-constrained scientific workflow in multi-cloud environments, referred to as MinCost-MC, which is shown to be NP-complete. Within a generic three-layer workflow execution framework, we propose a scientific Workflow Mapping algorithm for Financial Cost Optimization, referred to as WMFCO. The key idea of this algorithm is to classify workflow modules for VM type selection and then strategically choose suitable VM types, physical machines, and network bandwidths to ensure that the deadline is met. Extensive simulation results show that WMFCO exhibits a superior performance over existing algorithms in terms of financial cost in multi-cloud environments.

The rest of this paper is organized as follows. Section 2 conducts a survey of related work on workflow mapping. Section 3 constructs the model of workflow execution in multiple clouds and defines MinCost-MC. Section 4 designs the workflow mapping algorithm and Section 5 evaluates the performance based on simulations. In Section 6, we conclude our work.

\section{RELATED WORK}

Workflow scheduling has been the focus of research in the past decade[5]. This section provides a brief description of related work on workflow scheduling under deadline and budget constraints. These problems have been well studied in different contexts such as distributed computing and its extension to clouds. Such efforts especially with cost awareness are summarized and compared in a recent survey conducted by Alkhanak et al. in [3, 4].

Generally, budget-constrained workflow scheduling aims to finish the execution of a workflow as quickly as possible under a budget constraint. There exist a significant number of efforts on workflow scheduling within a specified budget in grids, such as[19, 21, 22, 25]. Particularly, in[22], Sakellariou et al. proposed two methods to schedule workflows in grids, i.e., LOSS and GAIN. For a DAG-structured workflow (HEFT/HBMCT), they started with a minimal cost schedule, and then rescheduled the workflow under a specified budget constraint to minimize the overall execution time.

On the other hand, deadline-constrained workflow scheduling aims to complete the execution of a workflow with the lowest possible cost within a specified deadline. Similarly, many studies have been conducted on workflow mapping under deadline constraints. Yu et al. designed a cost-based workflow mapping strategy by partitioning modules and distributing deadlines to achieve the minimum cost within the given deadline[12]. They divided the total deadline into sub-deadlines based on module partitions, which are proportional to their minimum execution time. Abrishami et al. proposed a workflow scheduling scheme in terms of QoS and Partial Critical Path $(P C P)$ on SaaS clouds to minimize the financial cost of workflow execution without exceeding their deadline[2]. By leveraging some existing heuristics for critical path $(C P)$, they scheduled tasks on a critical path to minimize the financial cost under the specified deadline constraint. Each $P C P$ is formed after the critical task is processed and the deadline is set to be the start time of the processed critical task. This mapping process is repeated until all tasks have been scheduled. $\operatorname{In}[18]$, Sobhan et al. used Level Load Balancing to improve deadline distribution and lower communication cost.
Some efforts have been made on workflow mapping and resource allocation on clouds with focus on both performance and cost. In [17], Mao et al. made an investigation into automatic scale clouds under deadline and budget constraints and proposed a Scaling-ConsolidationScheduling (SCS) algorithm with VM instances as fundamental calculating elements. In[9], Hacker proposed a solution that combines four scheduling strategies according to an online estimate of physical resources usage. In[20], Rodriguez et al. designed a strategy to utilize resource allocation and workflow mapping on IaaS clouds to minimize the total financial cost within a specified deadline. Their method included an approach based on Particle Swam Optimization, without considering the cost of data transfer between different data centers. In[14], to execute multiple large-scale scientific workflows, Jiang et al. addressed two major challenges on multiple public clouds: execution coordination and resource allocation. They improved a pulling-based workflow management system named DEWE v2, which is based on a profiling resource allocating strategy, and validated their solution in terms of both deadline and cost. The Cost Effective Deadline Aware (CEDA) scheduling strategy proposed by Haidri et al. aims at optimizing the total execution time and cost within the deadline[10].

For workflow scheduling under multiple constraints, researchers have also explored various meta-heuristic methods such as guided random search including Genetic Algorithms (GA)[11, 13], Ant Colony Optimization (ACO)[7], and Particle Swarm Optimization (PSO)[20, 24].

Many existing efforts are focused on scientific workflow execution at a single data center/cloud site. With the availability of highspeed links to support big data transfer over wide-area networks, large-scale workflows have been increasingly deployed across multiple data centers or clouds, but the workflow scheduling problem remains largely unexplored. For VM assignment in multi-clouds, cloud service providers should optimize the usage of physical resources through a careful provisioning of VM instances and balance the trade-off between cost and performance. In[6], Byun et al. proposed a workflow algorithm, Partitioned Balanced Time Scheduling (PBTS), to minimize the overall cost of execution within a given deadline. Their algorithm estimates the minimum number of instances required to minimize the financial cost. In [16], Malawski et al. proposed three algorithms to schedule a cluster of scientific workflows on IaaS clouds. Their algorithms were designed to maximize the number of workflows that could be completed whithin specified budget and deadline constraints. Nevertheless, most of these algorithms including PBTS consider only one instance type while many commercial cloud service providers nowadays provide multiple VM types for users to choose from. In this paper, different from the aforementioned work on workflow mapping, we study a more general workflow mapping problem that considers data storage, $I / O$ operations, and the provisioning of different VM types.

\section{MATHEMATICAL MODELS AND PROBLEM DEFINITION}

In this section, we consider a three-layer scientific workflow execution framework to model and analyze workflow execution on VMs provisioned on physical machines (PMs) deployed on multiple clouds. 


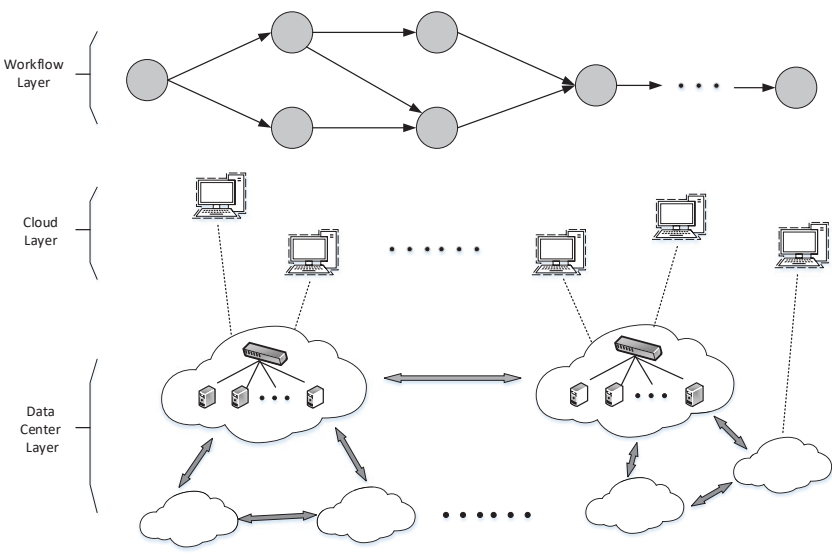

Figure 1: The workflow execution framework in multiple clouds.

\subsection{A Scientific Workflow Execution Framework}

As illustrated in Figure 1, the workflow execution framework is composed of three parts, namely, workflow, cloud, and data center.

3.1.1 Workflow Layer. In this layer, we consider a workflow structure, which is comprised of a set of computing modules with execution dependency and inter-module data transfer.

Such a workflow is typically modeled as a Directed Acyclic Graph (DAG) $G_{w}=\left(V_{w}, E_{w}\right)$, consisting of $\left|V_{w}\right|$ modules with $\left|E_{w}\right|$ directed edges $e_{i, j}$ denoting the data dependency between modules $w_{i}$ and $w_{j}$. We use $W L\left(w_{i}\right)$ to denote the workload of module $w_{i}$ and use $D S_{i, j}$ to denote the data size transferred over edge $e_{i, j}$.

3.1.2 Cloud Layer. In this layer, we consider a cloud network of VMs provisioned on various PMs.

We describe the set of VM types as $V T=\left\{v t_{0}, v t_{1}, \ldots, v t_{n-1}\right\}$, each of which specifies three performance attributes, i.e., CPU frequency, $I / O$ speed, and disk capacity. Based on these parameters, we consider a $V T$ pricing model $p(v t)=f\left(f_{C P U}, r_{I / O}, c_{d i s k}\right)$, which determines the financial cost per unit of time for using a VM instance of that particular type.

Note that the disk capacity of the selected VT $v t$ must be larger than the data size of a computing module that runs on it, i.e.,

$$
c_{d i s k}(v t) \geq\left\{\begin{array}{l}
D S_{i}(w)+D S_{o}(w), \text { if } D S_{i} \text { and } D S_{o} \text { coexist; } \\
\max \left(D S_{i}(w), D S_{o}(w)\right), \text { otherwise }
\end{array}\right.
$$

where $D S_{i}$ and $D S_{o}$ denote the input and output data size of a module, respectively.

3.1.3 Data Center Layer. This layer represents a collection of distributed data centers comprised of clusters of physical machines (PMs) and interconnected by high-performance networks. We model interconnected multi-cloud infrastructures as a complete weighted graph $G_{m c}=\left(V_{m c}, E_{m c}\right)$.

We use a set of $m$ available bandwidth type $B T=\left\{b t_{0}, b t_{1}, \ldots, b t_{m-1}\right\}$ to support inter-cloud data transfer and define a corresponding $B T$ pricing model based on the requested bandwidth,i.e., $p(d t)=f(b t)$.
Following a large body of existing work in a single cloud, we ignore the financial cost of intra-cloud data transfer due to its relative insignificance compared with computing itself.

\subsection{Time Execution Models}

3.2.1 Time of Module Execution. For a computing module $w$, the execution time may involve multiple code segments and can be affected by both CPU speed and I/O speed. We model the program execution time $T_{C P U}$ and input-output time $T_{I / O}$ as

$$
\begin{gathered}
T_{C P U}=\frac{W L(w)}{f_{C P U}(v t)}, \\
T_{I / O}=\frac{D S_{i}(w)+D S_{o}(w)}{r_{I / O}(v t)} .
\end{gathered}
$$

Obviously, the total module execution time $T_{m e}$ on an allocated VM instance is calculated as

$$
T_{m e}(w)=T_{C P U}+T_{I / O} .
$$

3.2.2 Time of Data Transfer. The data transfer time between two clouds could be estimated by dividing the transfer data size by the selected bandwidth type $b t$, i.e.,

$$
T_{d t}\left(e_{w}\right)=\frac{D S_{i, j}}{b t} .
$$

3.2.3 Workflow Makespan. The makespan MS of a workflow $G_{w}$ depends on the critical modules on the critical path $C P$, which is the longest execution path. The $M S$ of a workflow execution process is calculated as

$$
M S=\sum_{\text {for all } w \in C P}\left(T_{m e}(w)+T_{w a i t}(w)\right)+\sum_{\text {for all } e_{w} \in C P} T_{d t}\left(e_{w}\right) .(6)
$$

3.2.4 Shortest Execution Time. The shortest execution time SET of a workflow $G_{w}$ is determined by the shortest path $(S P)$, which is the path of the shortest execution time among all paths from the start module to the end module. The SET of a workflow execution process is calculated as

$$
\text { SET }=\min _{\text {all possible paths }} T_{P},
$$

where $T_{P}$ is the execution time of each possible path.

\subsection{Financial Cost Models}

The total financial cost $C_{\text {Total }}$ of a workflow execution process is calculated as

$$
C_{\text {Total }}=C_{\text {Total }}(V M)+C_{\text {Total }}(d t),
$$

where $C_{\text {Total }}(V M)$ is the cost of each module running on a selected VM type $v t$, and $C_{\text {Total }}(d t)$ is the cost of data transfer between clouds using a bandwidth type $b t \in B T$ over an inter-cloud network link. Their detailed calculations are provided as follows:

$$
\begin{gathered}
C_{\text {Total }}(V M)=\sum_{\text {for all } w \in V_{w}}\left(T_{m e}(w, v t)+T_{\text {wait }}(w, v t)\right) \cdot p(v t), \text { (9) } \\
C_{\text {Total }}(d t)=\sum_{\text {for all } e_{w} \in E_{w}} T_{d t}\left(e_{w}, b t\right) \cdot p(d t)
\end{gathered}
$$

For convenience of reference, we tabulate in Table 1 a set of main notations used in the models. 
Table 1: List of notations used in the cost models.

\begin{tabular}{|l|l|}
\hline Parameters & Definitions \\
\hline$G_{w}\left(V_{w}, E_{w}\right)$ & a computing workflow \\
\hline$e_{i, j}$ & the edge of dependency from module $w_{i}$ to $w_{j}$ \\
\hline$V T$ & a set of available VM types \\
\hline$v t$ & a VM type \\
\hline$G_{m c}\left(V_{m c}, E_{m c}\right)$ & a multi-cloud network graph \\
\hline$B T$ & a set of inter-cloud bandwidth types \\
\hline$b t$ & a bandwidth type \\
\hline$D$ & the specified deadline \\
\hline$T_{C P U}$ & the CPU bound program execution time \\
\hline$T_{I / O}$ & the I/O bound program execution time \\
\hline$T_{m e}$ & the overall module execution time \\
\hline$T_{d t}$ & the time of data transfer between clouds \\
\hline$T_{w a i t}$ & the module waiting time \\
\hline$M S$ & the makespan of a workflow \\
\hline$S E T$ & the shortest execution time of a workflow \\
\hline$M$ & a workflow mapping strategy \\
\hline
\end{tabular}

\subsection{Problem Formulation}

We define a workflow mapping strategy $\mathcal{M}$ as

$$
\mathcal{M}: v_{w} \rightarrow V M(v t, P M), \text { for all } w \in V_{w},
$$

where $V M(v t, P M)$ denotes a VM of type $v t$ provisioned from PM. Based on the above models, we define the following problem.

Definition 1. Workflow Mapping under Deadline Constraints to Minimize Cost in Multi-Clouds (MinCost-MC).

Given:

$$
\begin{gathered}
G_{w}=\left(V_{w}, E_{w}\right), \\
G_{m c}=\left(V_{m c}, E_{m c}\right), \\
V T=\left\{v t_{0}, v t_{1}, \ldots, v t_{n-1}\right\}, \\
B T=\left\{b t_{0}, b t_{1}, \ldots, b t_{m-1}\right\}, \\
\text { and a specified deadline } D,
\end{gathered}
$$

Objective: to design a workflow scheduling strategy $\mathcal{M}$ to achieve the Minimal Financial Cost (MinFC), i.e.,

$$
\text { MinFC }=\min _{\text {all possible } M} C_{\text {Total }},
$$

while satisfying the following specified deadline for completion:

$$
M S \leq D .
$$

\subsection{Complexity Analysis}

For any given schedule, we can always verify in polynomial time if it is a feasible schedule by calculating and comparing the makespan with the given deadline, so it is obvious that MinCost-MC $\in \mathrm{NP}$. We prove the NP-hardness of MinCost-MC by showing that a classical NP-complete problem, Multiple-Choices Knapsack Problem (MCKP), is a special case of MinCost-MC.

We consider a special case of MinCost-MC-Pipeline where the workflow consists of a linear pipeline of $m$ modules without data transfer time over each edge. We first compute the execution time of module $w$ on a virtual machine of each VM type $v t$ and the corresponding execution cost $C(w)$. The goal is to minimize the total financial cost $C_{\text {Total }}$ of the pipeline-structured workflow within a given deadline $D$. We show that MinCost-MC-Pipeline is essentially the MCKP problem: $m$ modules in MinCost-MC-Pipeline correspond to $m$ classes in MCKP, and $n$ available VM types for each module $w$ correspond to $n$ items in each class in MCKP. We set the value of capacity $c=D$ and assign each item in a class in MCKP a weight, which is equivalent in value to the corresponding makespan in MinCost-MC-Pipeline, and a profit, which is equivalent in value to $K-C_{\text {Total }}$ in MinCost-MC-Pipeline, where $K$ is a sufficiently large constant such that $K \geq C_{\text {Total }}$. Under this construction, MCKP and MinCost-MC-Pipeline are the same problem that chooses exactly one item or VM type per class or module to maximize the total profit, subject to the knapsack capacity or deadline constraint $D$. We ignore the data transfer in MinCostMC-Pipeline, so $C_{\text {Total }}$ is simply the sum of financial cost of all module executions and the module dependency along the pipeline does not affect the problem. Since a special case is NP-complete, the original MinCost-MC problem is also NP-complete.

\section{ALGORITHM DESIGN}

We design a Workflow Mapping for Financial Cost Optimizing (WMFCO) algorithm to solve MinCost-MC, as shown in Alg. 1.

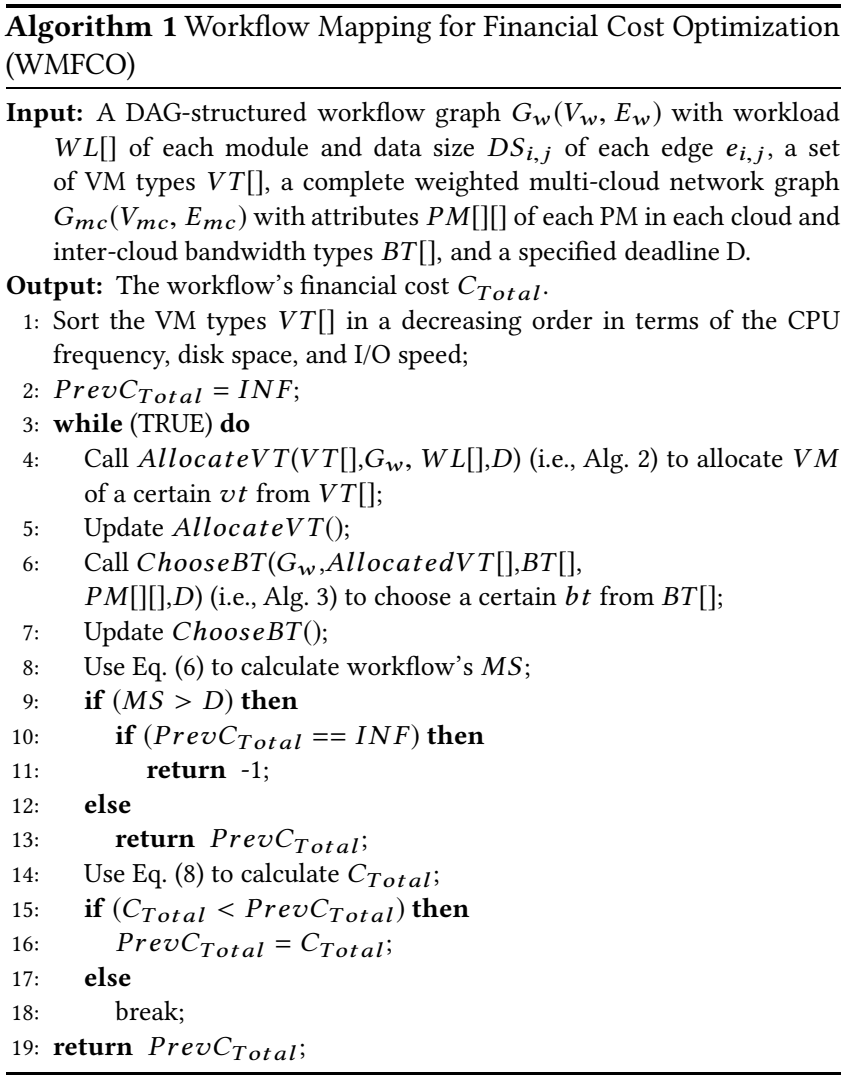

\section{Step 1: Sort VMs and classify modules}

1) Sort the VMs by their types $V T[]$ in a decreasing order in terms of the CPU frequency, disk space and $I / O$ speed.

2) For each module, allocate the best available VM of a certain type $v t$ that satisfies the minimal storage requirement defined in 
Eq. (1), and calculate the execution time of every module allocated on the VM, using Eq. (4).

3) Call Alg. 2 AllocateVT, which calls Function FindCP() in [23] and Find $S P()$ in Alg. 4 to obtain a critical path $(C P)$ and a shortest path $(S P)$ based on the module execution time. According to $C P$ and $S P$, the modules are divided into two categories: class $A$ is a set of modules along the path $S P$ except those belonging to $C P$ if two paths are module-joint and class $B$ contains the rest. Note that Function FindSP() is a direct application of Dijkstra's Algorithm [8] considering the weight of both modules and edges.

In Figure 2, we provide a simple example to explain the classification process, using a scientific workflow composed of 9 modules $w_{1}, w_{2}, \ldots, w_{9}$ with a start module $w_{0}$ and an end module $w_{10}$ (as shown in yellow) denoting the data input and output process of a scitific workflow. After assigning VMs for modules and bandwidths for inter-cloud links, we calculate the total module execution time and data transfer time to compute $C P$ and $S P$. For simplicity, we label the data transfer time on each directed edge as well as the execution time of each computing module. As shown in Table 2, there are 6 paths.

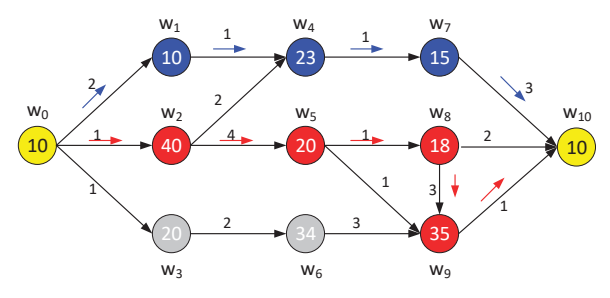

Figure 2: The workflow used in the example.

Table 2: The weight of each path.

\begin{tabular}{|c|c|c|}
\hline Index & Path & Weight \\
\hline$P_{1}$ & $\left\{w_{0}, w_{1}, w_{4}, w_{7}, w_{10}\right\}$ & 65 \\
\hline$P_{2}$ & $\left\{w_{0}, w_{2}, w_{4}, w_{7}, w_{10}\right\}$ & 105 \\
\hline$P_{3}$ & $\left\{w_{0}, w_{2}, w_{5}, w_{8}, w_{10}\right\}$ & 106 \\
\hline$P_{4}$ & $\left\{w_{0}, w_{2}, w_{5}, w_{9}, w_{10}\right\}$ & 121 \\
\hline$P_{5}$ & $\left\{w_{0}, w_{2}, w_{5}, w_{8}, w_{9}, w_{10}\right\}$ & 143 \\
\hline$P_{6}$ & $\left\{w_{0}, w_{3}, w_{6}, w_{9}, w_{10}\right\}$ & 116 \\
\hline
\end{tabular}

By calculating the execution time of each path, we know that $P_{5}$ is the critical path (as shown with red arrows), and $P_{1}$ is the shortest path (as shown with blue arrows). Therefore, blue modules belong to class $A$, and the other modules belong to class $B$ in this case.

\section{Step 2: Allocate appropriate VM}

We check if the makespan of the workflow exceeds the specified deadline: a) if it exceeds the deadline, there is no feasible mapping strategy; b) otherwise, downgrade the VM types for the modules in class $A$ to the next lower VM types until the makespan exceeds the deadline or the VMs in class $A$ have been downgraded to the worst type. If the makespan is within the deadline, then downgrade the VM types for the other modules in class $B$ using the same procedure as the modules in class $A$.

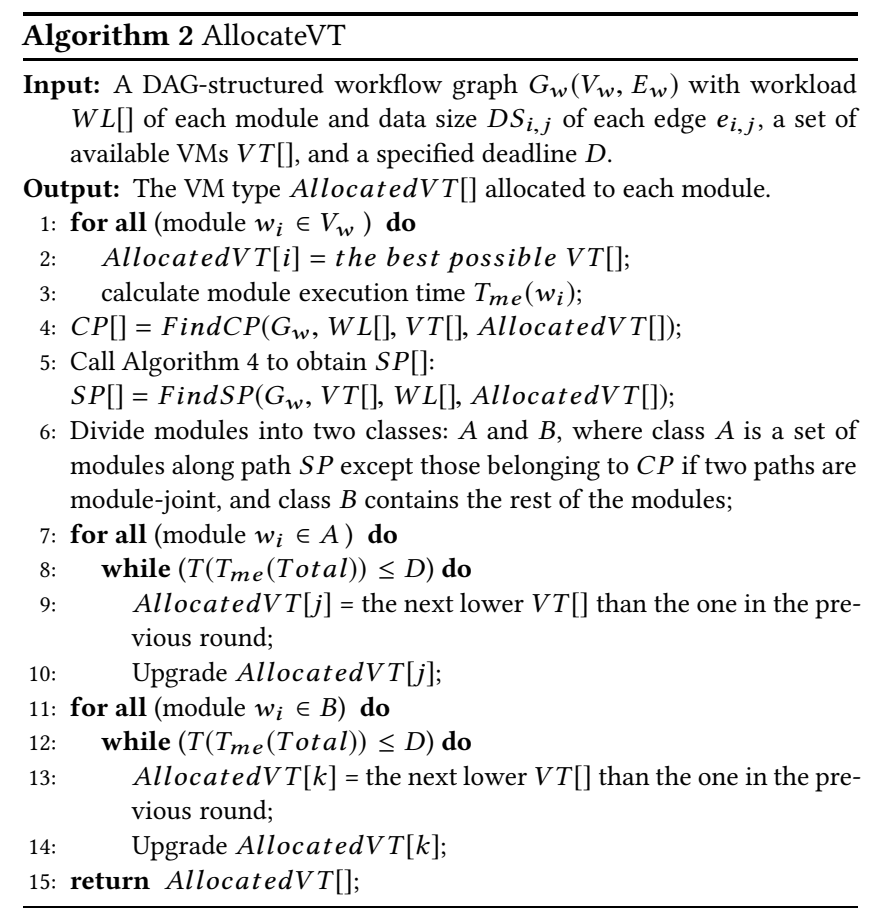

Step 3: Choose appropriate bandwidth and PM

1) In Alg. 3 ChooseBT, select the PM in a data center to provision the corresponding VM. To avoid inter-cloud data transfer, provision VM instances on the same PM or different PMs in the same cloud wherever possible. If they cannot be provisioned from the same cloud due to resource constraints, then move the VM instances to another cloud.

2) Allocate the largest bandwidth type $b t$ to the corresponding inter-cloud network link to minimize the data transfer time, if the inter-cloud data transfer is unavoidable.

3) If a scheduling strategy $\mathcal{M}$ is feasible, calculate the makespan $M S$ of the workflow and check if it exceeds the deadline $D$ : a) if it exceeds the deadline, continuously upgrade the VM type for every computing module until the mapping strategy becomes valid; b) otherwise, continuously downgrade the bandwidth type until the workflow makespan exceeds the specified deadline and then return the last valid workflow mapping scheme.

Step 4: Calculate the total financial cost

Calculate the workflow's total financial $\operatorname{cost} C_{\text {Total }}$. If the financial cost of the current mapping strategy $\mathcal{M}$ is less than the previous cost, return to Step 1 and continue using Find $C P()$ and Find $S P()$ to compute a new $C P$ and a new $S P$, respectively; otherwise, terminate the mapping procedure.

In Alg. 2, there are three "for" loops, and the time complexity is $O(m \cdot n)$, where $m$ is the number of modules and $n$ is the number of available VM types. In Lines 4-5, we use modules and edges to find $C P$ and $S P$ with time complexity of $O\left(m \cdot\left|E_{w}\right|\right)$ and $O\left(m^{2}\right)$, respectively. In Alg. 3, the time complexity of Lines 1-3 is of $O\left(\left|E_{w}\right|^{2}\right)$, and that of Lines 4-20 is of $O\left(\left|V_{m c}\right|^{2} \cdot\left|E_{w}\right| \cdot m\right)$. Hence, the overall time complexity is $O\left(\max \left(m \cdot\left|E_{w}\right|, m \cdot n, m^{2}\right)+\max \left(\left|E_{w}\right|^{2},\left|V_{m c}\right|^{2} \cdot\left|E_{w}\right|\right.\right.$. $m)$ ), where $\left|E_{w}\right|$ is the number of edges and $\left|V_{m c}\right|$ is the number of data centers. 

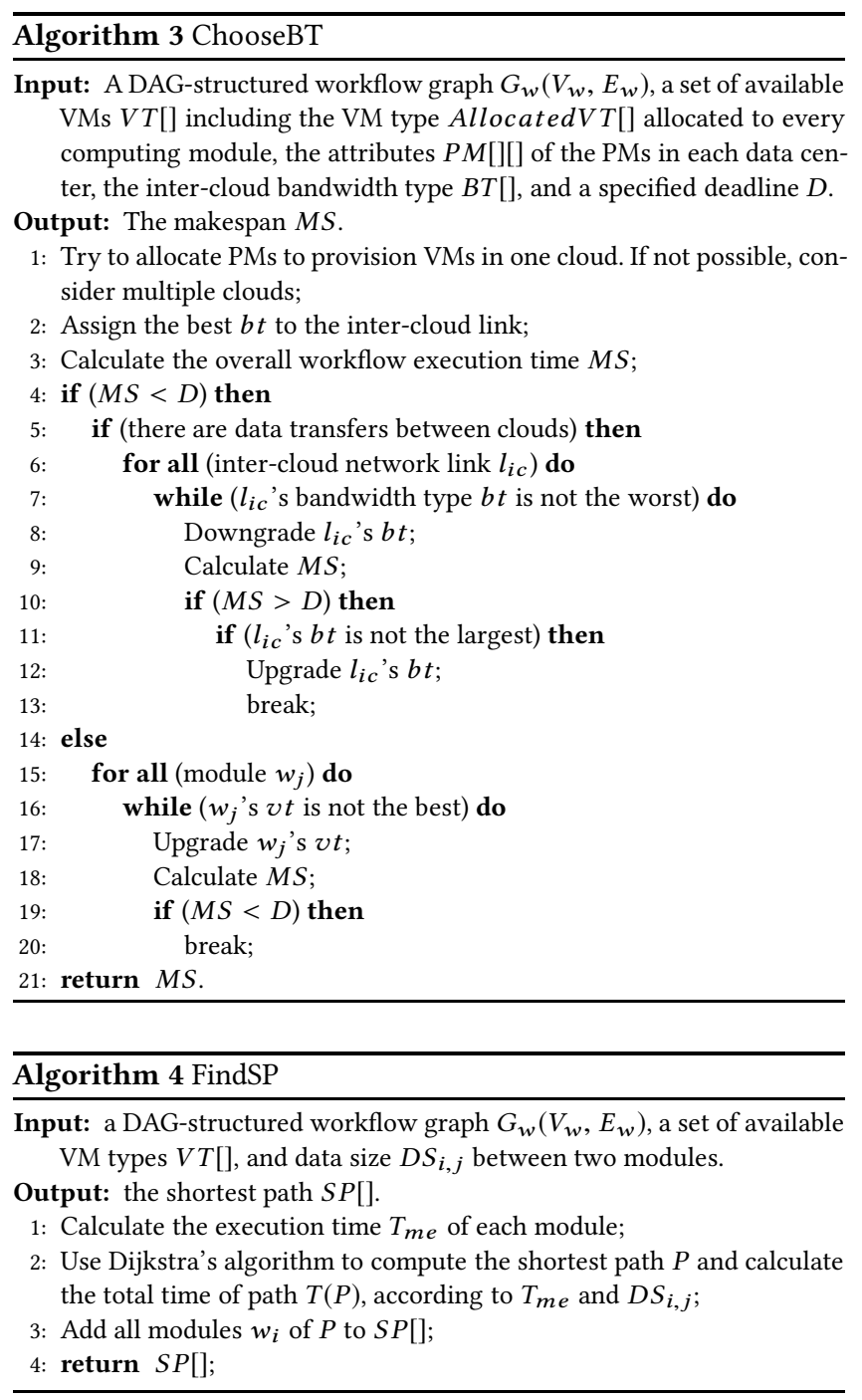

\section{ALGORITHM IMPLEMENTATION AND PERFORMANCE EVALUATION}

In this section, we conduct simulations within a three-layer workflow execution framework, and evaluate the cost and performance of the proposed mapping strategy in multi-clouds.

\subsection{Simulation Settings}

We compare the proposed algorithm with two exiting algorithms, i.e., Max-min[15] and MCWM[23], as briefly introduced below, for cost and performance evaluation.

1) Max-min: Max-min calculates the earliest completion time for each module on any available machine. It first schedules large modules and maps them to the corresponding machines. Then it deploys the modules in the data centers at random, and allocates the best bandwidth for data transfer between clouds. If the execution time exceeds the deadline, it would downgrade the bandwidth type until the mapping scheme becomes valid.
2) MCWM: MCWM was designed to optimize the performance of workflows under budget constraints in multi-cloud environments and is one of the state-of-the-art solutions suitable for comparison.

We first set a sufficiently large budget to ensure that the makespan $M S$ of the workflow can meet the specified deadline. We then adopt a binary search method by halving the budget until MS exceeds the deadline. The median between the current budget and the previous budget is selected as the budget, which satisfies the deadline.

We consider a set of workflow mapping problems of different scales to evaluate the performance. We define the problem scale as a 4-tuple $\left(m,\left|E_{w}\right|, n, c\right)$, where $m$ is the number of computing modules, $\left|E_{w}\right|$ is the number of directed edges, $n$ is the number of available VM types, and $c$ is the number of data centers. The parameters of each $\mathrm{PM} / \mathrm{VM}$ as well as the link bandwidths between data centers are set to be appropriate values according to the common hardware configuration and network infrastructure. Specifically, in each problem instance, the CPU frequency of each core is set within a range from $1 \mathrm{GHz}$ to $4 \mathrm{GHz}$, the I/O speed is set within a range from $20 \mathrm{Mbps}$ to $300 \mathrm{Mbps}$, the disk capacity is set within a range from $500 \mathrm{~GB}$ to $4 \mathrm{~TB}$, and the network bandwidth is set within a range from $1 \mathrm{Gbps}$ to $10 \mathrm{Gbps}$.

We generate workflow instances of various sizes as follows:

a) Lay out all modules sequentially from left to right. For each module, select one preceding module at random among the modules on its left and select one succeeding module at random among the modules on its right. Note that the first module is considered as the source of the entire workflow and hence only requires one succeeding module, and the last module is considered as the sink of the entire workflow and hence only requires one preceding module;

b) Repeatedly select two modules at random and add a directed edge between them from left to right until achieving the specified number of links;

c) Each module is assigned a random computing workload and each edge is assigned a random data size within the range $[5,500]$, in unit of million instructions and GBytes, respectively.

We compare WMFCO with Max-min and MCWM in terms of the overall cost of workflow execution under the same deadline. We define the MinFC performance improvement of MinCost-MC over the other algorithms as:

$$
\operatorname{Imp}(\text { Other })=\frac{\text { MinFC }_{\text {other }}-\text { MinFC }_{W M F C O}}{\text { MinC }_{\text {other }}} \times 100 \%,
$$

where $M i n F C_{\text {other }}$ is the cost of MinFC obtained by the other two algorithms, i.e., Max-min, MCWM, and MinFC $W M F C O$ is the cost of MinFC obtained by WMFCO.

\subsection{Simulation Results}

We consider 20 different problem scales from small to large, indexed from 1 to 20 , respectively. For every problem scale $\left(m,\left|E_{w}\right|, n, c\right)$, we generate 20 problem instances at random. For each problem instance, we select 10 deadline levels with the same interval of $\Delta D=$ $\left(D_{\max }-D_{\min }\right) / 10$ within a certain deadline range $\left[D_{\min }, D_{\max }\right]$. 
In Table 3, we provide the average MinFC improvement percentage as well as the standard deviation obtained by WMFCO over Max-min and MCWM in each of these 20 problem sizes. These performance measurements are also plotted in Figure 3 for a visual comparison.

Table 3: The average MinFC improvement percentages $\alpha=$ $\operatorname{Imp}(\mathbf{M a x}-\mathbf{m i n})$ and $\beta=\operatorname{Imp}(\mathbf{M C W M})$ of WMFCO at different problem scales.

\begin{tabular}{|c|c|cc|cc|}
\hline Problem Level & $\left(m,\left|E_{w}\right|, n, c\right)$ & $\alpha$ & StdDv & $\beta$ & StdDv \\
\hline 1 & $(5,7,5,5)$ & 54.27 & 10.31 & 11.30 & 6.48 \\
2 & $(7,13,7,8)$ & 48.13 & 16.01 & 10.02 & 5.17 \\
3 & $(9,22,9,11)$ & 51.31 & 15.44 & 10.54 & 5.12 \\
4 & $(11,32,11,14)$ & 48.32 & 13.27 & 10.47 & 4.41 \\
5 & $(13,45,13,17)$ & 49.00 & 12.12 & 11.10 & 4.51 \\
6 & $(15,60,15,20)$ & 54.07 & 12.89 & 12.00 & 4.91 \\
7 & $(17,76,17,23)$ & 50.72 & 13.32 & 10.12 & 6.06 \\
8 & $(19,94,19,26)$ & 48.39 & 18.68 & 8.68 & 5.41 \\
9 & $(21,115,21,29)$ & 51.77 & 10.12 & 10.93 & 5.61 \\
10 & $(23,137,23,31)$ & 54.81 & 15.01 & 8.01 & 5.06 \\
11 & $(27,188,27,34)$ & 45.14 & 10.78 & 10.79 & 6.39 \\
12 & $(31,247,31,37)$ & 52.46 & 17.93 & 7.94 & 5.27 \\
13 & $(35,314,35,40)$ & 51.32 & 18.84 & 8.95 & 5.46 \\
14 & $(39,389,39,43)$ & 52.55 & 14.59 & 10.99 & 5.59 \\
15 & $(43,472,43,46)$ & 48.79 & 18.61 & 9.51 & 4.31 \\
16 & $(47,563,47,49)$ & 50.26 & 11.25 & 10.36 & 5.39 \\
17 & $(51,662,51,52)$ & 48.69 & 10.56 & 10.56 & 5.31 \\
18 & $(55,769,55,55)$ & 45.20 & 16.89 & 6.93 & 3.99 \\
19 & $(59,884,59,58)$ & 42.18 & 14.76 & 4.76 & 3.74 \\
20 & $(63,1007,63,61)$ & 43.85 & 13.58 & 3.59 & 2.81 \\
\hline
\end{tabular}

Similarly, we consider 10 different deadline levels from short to long, indexed from 1 to 10 , respectively, at each of which, we repeat the mapping simulation across 20 different problem scales. In Table 4, we provide the average MinFC improvement percentage as well as the standard deviation obtained by WMFCO over Max-min and MCWM at each of these 10 deadline levels. These performance measurements are also plotted in Figure 4 for a visual comparison.

Table 4: The average MinFC improvement percentages $\alpha=$ $\operatorname{Imp}(\mathbf{M a x}-\mathbf{m i n})$ and $\beta=\operatorname{Imp}(\mathbf{M C W M})$ of WMFCO at different deadline levels.

\begin{tabular}{|c|cc|cr|}
\hline Deadline Level & \multicolumn{1}{|c|}{$\alpha$} & StdDv & $\beta$ & StdDv \\
\hline 1 & 48.91 & 9.18 & 8.74 & 3.96 \\
2 & 49.45 & 8.01 & 8.27 & 5.48 \\
3 & 47.81 & 11.44 & 10.27 & 4.41 \\
4 & 49.91 & 10.09 & 9.82 & 5.09 \\
5 & 48.41 & 11.04 & 9.66 & 4.78 \\
6 & 50.82 & 11.52 & 11.52 & 4.19 \\
7 & 50.17 & 10.11 & 9.61 & 4.66 \\
8 & 50.86 & 8.53 & 8.81 & 4.07 \\
9 & 50.13 & 9.06 & 8.56 & 3.62 \\
10 & 51.15 & 15.42 & 7.08 & 4.91 \\
\hline
\end{tabular}

For a holistic visual comparison, we plot all cost improvement percentages of WMFCO over Max-min and MCWM in Figure 5 and

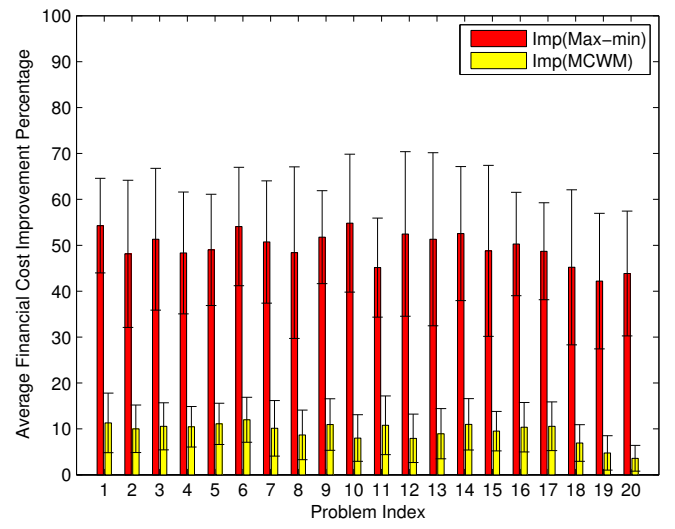

Figure 3: The average $M i n F C$ improvement percentage across 200 instances (20 random scientific workflow instances $\times 10$ deadline levels) in each problem size.

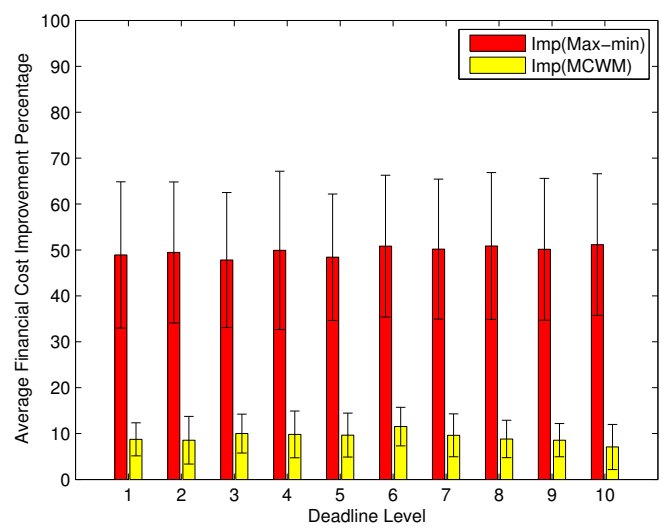

Figure 4: The average $M i n F C$ improvement percentage across 400 instances $(20$ problem scales $\times 20$ random scientific workflow instances) at each deadline level.

Figure 6, respectively. In the 3D figure, each point denotes the average $\mathrm{MinC}$ improvement percentage across 20 problem instances of the same problem scale at the same deadline level. Note that actual deadlines would differ in various problem instances. In these performance measurements, we observe that WMFCO achieves about $50 \%$ improvement over Max-min on average and about $9 \%$ improvement over MCWM on average.

\section{CONCLUSION}

We formulated a workflow scheduling problem under deadline constraints to minimize the financial cost of scientific workflows in multi-cloud environments, referred to as MinCost-MC. We showed it to be NP-complete and proposed a heuristic algorithm that considers module execution time and inter-cloud data transfer in workflow scheduling. We conducted extensive simulations and illustrated 


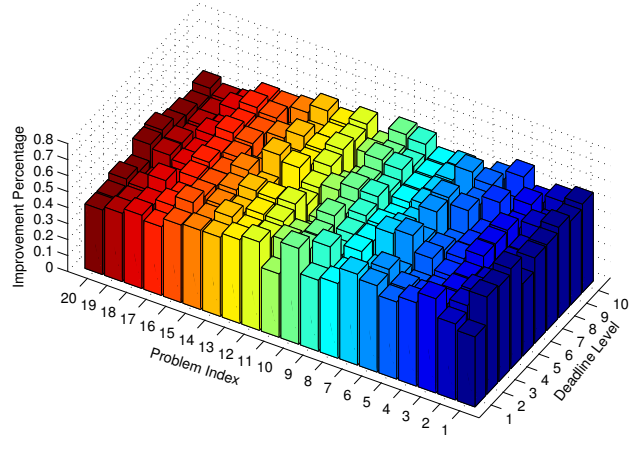

Figure 5: The overall MinFC improvement percentage of WMFCO over Max-min with various deadline levels and problem sizes.

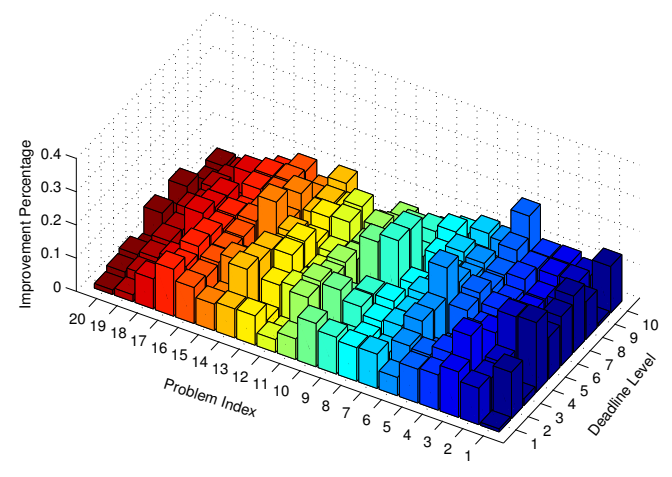

Figure 6: The overall $M i n F C$ improvement percentage of WMFCO over MCWM with various deadline levels and problem sizes.

the performance superiority of the proposed algorithm over other existing methods.

It is of our interest to refine and generalize the mathematical models for more accurate modeling and estimation of workflow execution in real-life clouds. For instance, in a real cloud system, physical devices (servers and links) may be subject to a certain probability of failure. We will improve our mapping strategy by taking into account multiple practical factors in our predictive model.

\section{ACKNOWLEDGMENT}

This research is sponsored by the National Key R\&D Program of China under Grant No. 2017YFB1300301, National Nature Science Foundation of China under Grant No. 61472320 and U1609202, and Key Research and Development Plan of Shaanxi Province under Grant No. 2018GY-011 with Northwest University, China, and also by U.S. National Science Foundation under Grant No. CNS-1828123 with New Jersey Institute of Technology.

\section{REFERENCES}

[1] 2018. Amazon EC2. http://aws.amazon.com/ec2/.

[2] S. Abrishami and M. Naghibzadeh. 2012. Deadline-constrained workflow scheduling in software as a service Cloud. Scientia Iranica 19, 3 (2012), 680-689.

[3] E.N. Alkhanak, S.P. Lee, and S.U.R. Khan. 2015. Cost-aware challenges for workflow scheduling approaches in cloud computing environments: Taxonomy and opportunities. Future Generation Computer Systems 50, C (2015), 3-21.

[4] E.N. Alkhanak, S.P. Lee, R. Rezaei, and R.M. Parizi. 2016. Cost optimization approaches for scientific workflow scheduling in cloud and grid computing: A review, classifications, and open issues. Journal of Systems and Software 113 (2016), 1-26.

[5] M. Atkinson, S. Gesing, J. Montagnat, and I. Taylor. 2017. Scientific workflows: Past, present and future. Future Generation Computer Systems 75 (2017), 216227

[6] E.K. Byun, Y.S. Kee, J.S. Kim, and S. Maeng. 2011. Cost optimized provisioning of elastic resources for application workflows. Future Generation Computer Systems 27, 8 (2011), 1011-1026.

[7] W. Chen and J. Zhang. 2009. An Ant Colony Optimization Approach to a Grid Workflow Scheduling Problem With Various QoS Requirements. IEEE Transactions on Systems,Man, and Cybernetics 39, 1 (2009), 29-43.

[8] E.W. Dijkstra. 1959. A note on two problems in connexion with graphs. Numer. Math. 1, 1 (1959), 269-271

[9] T.J. Hacker and K. Mahadik. 2011. Flexible resource allocation for reliable virtual cluster computing systems. In High PERFORMANCE Computing, Networking, Storage and Analysis. 1-12.

[10] R.A. Haidri, C.P. Katti, and P.C. Saxena. 2017. Cost Effective Deadline Aware Scheduling Strategy for Workflow Applications on Virtual Machines in Cloud Computing. Computer and Information Sciences (2017).

[11] Y. Jia and R. Buyya. 2006. Scheduling scientific workflow applications with deadline and budget constraints using genetic algorithms. Scientific Programming 14, 3-4 (2006), 217-230

[12] Y. Jia, R. Buyya, and K.T. Chen. 2005. Cost-Based Scheduling of Scientific Workflow Application on Utility Grids. In International Conference on E-Science and Grid Computing. 8 pp.-147.

[13] Y. Jia, M. Kirley, and R. Buyya. 2007. Multi-objective planning for workflow execution on Grids. In Ieee/acm International Conference on Grid Computing. 1017.

[14] Q. Jiang, Y.C. Lee, and A.Y. Zomaya. 2015. Executing Large Scale Scientific Workflow Ensembles in Public Clouds. In International Conference on Parallel Processing. 520-529.

[15] M. Maheswaran, S. Ali, H.J. Siegel, D. Hensgen, and R.F. Freund. 1999. Dynamic Mapping of a Class of Independent Tasks onto Heterogeneous Computing Systems. F. Parallel and Distrib. Comput. 59, 2 (1999), 107-131.

[16] M. Malawski, G. Juve, E. Deelman, and J. Nabrzyski. 2015. Cost- and deadlineconstrained provisioning for scientific workflow ensembles in IaaS clouds. Future Generation Computer Systems 48, 1 (2015), 1-18.

[17] M. Mao and M. Humphrey. 2011. Auto-scaling to minimize cost and meet application deadlines in cloud workflows. In High PERFORMANCE Computing, Networking, Storage and Analysis. 1-12.

[18] S. Omranian-Khorasani and M. Naghibzadeh. 2017. Deadline constrained load balancing level based workflow scheduling for cost optimization. In IEEE International Conference on Computational Intelligence and Applications. 113-118.

[19] M. Rahman, S. Venugopal, and R. Buyya. 2008. A Dynamic Critical Path Algorithm for Scheduling Scientific Workflow Applications on Global Grids. In $e$-Science and Grid Computing, IEEE International Conference on. 35-42.

[20] M.A. Rodriguez and R. Buyya. 2014. Deadline Based Resource Provisioningand Scheduling Algorithm for Scientific Workflows on Clouds. Cloud Computing IEEE Transactions on 2, 2 (2014), 222-235.

[21] R. Sakellariou and H. Zhao. 2004. A hybrid heuristic for DAG scheduling on heterogeneous systems. Proc.intl Par.distr.proc.symp (2004), 111

[22] R. Sakellariou, H. Zhao, E. Tsiakkouri, and M.D. Dikaiakos. 2007. Scheduling Workflows with Budget Constraints. Springer, Boston, MA. 189-202 pages.

[23] C.Q. Wu and H. Cao. 2016. Optimizing the Performance of Big Data Workflows in Multi-cloud Environments Under Budget Constraint. In IEEE International Conference on Services Computing. 138-145.

[24] Z. Wu. 2013. A market-oriented hierarchical scheduling strategy in cloud workflow systems. Fournal of Supercomputing 63, 1 (2013), 256-293.

[25] J. Yu and R. Buyya. 2006. A budget constrained scheduling of workflow applications on utility Grids using genetic algorithms. In Workflows in Support of Large-Scale Science, 2006. WORKS '06. Workshop on. 1-10. 